\title{
Nurturing sensemaking of, through, and with a mathematical model
}

\author{
Shulamit Kapon and Maayan Schvartzer
}

\author{
Faculty of Education in Science and Technology, Technion - Israel Institute of Technology, Haifa, Israel
}

\begin{abstract}
Students of physics, even at the undergraduate level, often perceive common sense and the use of mathematical formalisms in problem solving as disconnected activities. We present an ethnographic account of a case study, a year-long research apprenticeship of an $11^{\text {th }}$ grade physics student. The analysis examined the development of the student's understanding a mathematical equation as a model for a physical phenomenon, his use of that mathematization as a tool and as an object for sense making, and how these were nurtured by the mentor. Our analysis suggests that this process can be paralleled to the development of reading comprehension and that it involves the deciphering of the explicit and implicit meaning of the equation. We also show that using physics equations in this manner is not intuitive, and requires an epistemological change that needs to be necessitated for students.
\end{abstract}

Keywords: sensemaking, epistemology, mathematization, teaching, learning, inquiry

PACS: 01.40.Fk

\section{INTRODUCTION}

Studies of the history and philosophy of science point to "a shift where mathematical explanations came to be preferred to mechanical explanations when the latter did not conform to calculations." [1] Eminent physicists have long discussed the profound relationship between physics and mathematics. Heinrich Hertz for example is quoted in several books as saying [2]: "One cannot escape the feeling that these mathematical formulae have an independent existence and intelligence of their own, that they are wiser than we are, wiser even than their discoverers, that we get more out of them than was originally put into them."

A physics equation ("the formula") represents a concise and idealized description of a system using mathematical symbols and concepts which represent specific aspects of the system in question - it provides a model of the system [3]. What Hertz alluded to is that these equations not only afford the generation of explanations and predictions, but that they may have "hidden" unpacked meanings which, when uncovered, lead to insights about components, attributes and processes in the physical system that may not have been envisioned or grasped originally (e.g., the existence of a new particle). Hence an equation can function as a tool for making sense of the world, but also functions as an object for sensemaking in its own right.

A recently published review draws on a large body of literature to define sensemaking as "a dynamic process of building or revising an explanation in order to 'figure something out' to ascertain the mechanism underlying a phenomenon in order to resolve a gap or inconsistency in one's understanding. One builds this explanation out of a mix of everyday knowledge and formal knowledge by iteratively proposing and connecting up different ideas on the subject." [4]

Students of physics, even at the undergraduate level, often perceive common sense and the use of mathematical formalisms in problem solving as disconnected activities [5]. Moreover, when prompted to use formal notations (force diagrams) in problem solving, students' performance decreases [6]. Formal mathematical thinking does not seem to develop spontaneously $[7,8]$, unlike mechanistic reasoning, for example $[9,10]$. Students' modeling of a physical phenomenon with an equation and the use of that mathematization as a tool for sense making has mostly been examined in the context of problem solving sessions [11,12]. Research apprenticeships provide a richer context to examine this practice, since their disciplinary authenticity [13] is higher. Examining the interactions between the research mentor and the students around this mathematization, rather than focusing solely on students' engagement, can provide further insights, since high quality instruction makes features of particular content visible to, and learnable by students [14].

This paper presents a case study of a high school student research apprenticeship. It uses ethnographic 
account of the student's work and the interaction between the research mentor and the student to decipher the nature of understanding a mathematical equation as a model for a physical phenomenon, and the use of that mathematization as a tool and as an object for sense making. Understanding a mathematical equation as a model for a physical phenomenon is conceptualized here as analogous to reading comprehension.

\section{CONCEPTUAL FRAMEWORK}

Bruce Sherin [11] argued that physics expertise involves understanding what equations say in a fundamental sense, "a feeling" for the mathematical expression. He argued that this sense is informed by a complex knowledge system composed of a moderately large number of basic vocabulary elements, each associated with a simple "conceptual schema" consisting of an arrangement of symbols in an equation. For example, a "symbol template" such as $0=\square-\square$ is associated with a conceptual schema of canceling, implying that the expressions in each square cancel each another. Sherin termed these elements symbolic forms. Based on observations of undergraduate physics students engaged in problem solving he suggested a list of symbolic forms.

However, understanding "what equations say" goes beyond the acquisition of a list of related symbolic forms; in the same way that reading comprehension goes beyond independent, sequential development of hierarchically ordered lower-level skills such as phonological awareness, alphabetic understanding, and fluency. Advanced reading comprehension also requires higher order comprehension skills, background knowledge, and more [15].

Consider for example the equation for velocity in constant acceleration $\left(\mathrm{v}=\mathrm{v}_{0}+\mathrm{at}\right)$. We can think of the individual symbols, variables and parameters in the equation as words, the more complex components (at) as sentences, and the entire equation as a paragraph. We consider the conscious effort to figure out the explicit meaning of a mathematical model as sensemaking of the mathematization. A paragraph and complex sentences in a mathematical model also convey an implicit meaning that invites important inferences. We consider the conscious effort to understand the implicit meaning of this mathematization and its entailed insights as sensemaking through and with the formalism. This is the process that turns physics equations into a tool of inquiry, which leads to a better understanding of the phenomenon as well as the model, and can generate new knowledge. Productively engaging in this process is far more challenging for students than engaging in sensemaking of the model, though students need help in both. Learning to do so requires extensive and thoughtful support. In our observations we found that nurturing this process is one of the significant strengths of Mr. Shy Harazi, the research mentor in question.

\section{METHOD}

In the last few years, our research group has been conducting ethnographic studies in schools associated with the Acheret Center [16]. As part of their advanced physics studies in high school, all $11^{\text {th }}$ and $12^{\text {th }}$ grade students in the associated schools conduct an individual or paired long-term research project (18 months). The students carry out their research in the school laboratory, guided by physics teachers who act as research mentors and perceive themselves in this context as such $[13,17]$. Acheret associates Jewish and Arab schools. The specific project took place in a large school which serve mainly middle class secular Jewish families from the surrounding rural area. The research group follows the work of five experienced mentors. The unit of analysis is a mentor and his/her mentees and we observe them for the full duration of a project (1218 months). The data include videotapes of weekly working sessions (we attended working sessions with each mentor once every second or third week), the students' final research reports and the ongoing artifacts that they generate, stimulated recall interviews with the mentor and the students in which they are invited to recall and explain their concurrent thinking during that event, when prompted by a specific video or artefact, informal conversations with mentors and students documented as research notes, and observation of the K12 students' mock and final oral exams.

The project in the case study presented here is called "Waves." The student, an $11^{\text {th }}$ grade avid wave surfer, described the project in the introduction to his final report as follows:

/.../I'm interested in finding out when and why there will be good waves (a good wave is a wave that lasts for a long time, does not propagate too fast or too slowly, whose wall ${ }^{18}$ is about $80 \%$ of its amplitude ${ }^{18}$ height, and whose breaking is in an elliptical tube ${ }^{18}$ )/.../. Each of our experiments in this work derived from the preceding one: We started by examining the factors that influence wave velocity in a rope ((Exp. 1)), to be able to infer factors that influence wave velocity in water. We then studied how the depth of the water affects the wave velocity and the wavelength ((Exp. 2)), to be able to understand what would happen in the transition between different depths. In the next experiment, we tested how a one- step change in the depth of the water affected the wave velocity, wavelength, and amplitude ((Exp. 3)), to be able to hypothesise how a continuous slope ((i.e., 
continuous change of depth)) affects these wave features. In the last experiment, we examined how the wave features are influenced in a continuous slope of the aquarium floor ((i.e., continuous change of depth)) and the ratio between the amplitude and wavelength in which the wave breaks ((Exp. 4)).

Note that the student's use of the term "experiment" does not refer to one measurement, but rather to an iterative sequence of experimental design, measurements, and theoretical interpretations.

The data we discuss here are mainly related to Exp. 2 with some references to Exp. 1. We chose this phase of the work since it hallmarks a radical transition in the use of mathematization in the project.

\section{FINDINGS}

\section{A. A Radical Transition in the Use of Mathematization}

In Exp. 1, the hypothesis regarding the dependence between the velocity of the propagating wave, and the string's tension and its linear density was formulated through intuitive considerations complemented by dimension-analysis. The measurements confirmed this hypothesis. However, in Exp. 2 (water waves), relying solely on mechanistic reasoning and explanatory primitives $^{19}$, and a back-and-forth analogical mapping between waves in a string and waves in water did not result in a satisfactory explanation for the empirical results. At this point, the mentor suggested to the student "How do you feel about going to Wikipedia and asking it what it thinks about our experimental results?" (Lab, Jan 2, 2017, turn 319). This was when the student first encountered the formula for the phase velocity of a water wave (he refers to it as "the velocity of propagation") that later became the central mathematical model employed in different variations in the project (see Figure 1).

$$
\begin{aligned}
& v=\sqrt{\underbrace{\frac{g \lambda}{2 \pi}}_{1}+\underbrace{\left.\frac{\sigma 2 \pi}{\lambda \rho}\right) \tanh \left(\frac{2 \pi d}{\lambda}\right)}_{2}} \quad \begin{array}{l}
1 \text { - Gravity expression } \\
\text { 2-Surface tension expression } \\
\text { 3- Depth expression }
\end{array} \\
& \mathrm{g}-\text { Gravitational acceleration } \\
& \lambda \text {-Wavelength } \\
& \sigma \text {-Surface tension of the air-water interface } \\
& \rho \text {-Density of the water } \\
& \mathrm{d} \text { - Depth of the water }
\end{aligned}
$$

FIGURE 1. The basic mathematical model

The student related to this phase of the work in the stimulated recall interview about his final report:

275. Stu.: /.../And here, for the first time, I started using a logical explanation for my hunches and not just gut feelings.
Because usually, my hunches are correct. But, in this case, they weren't. And I realized I needed to express my hunches only if I have an explanation that relies on a formula or a theoretical model for why things happen.

The student re-evaluated in the interview his original intuitive hypothesis, which eventually turned out to be incorrect, as "not just a hunch". This "hunch" was based on strong explanatory primitives ${ }^{19}$. What we want to stress from our observations and the interview with the mentor is that the mentor knew before the student what the specific formula he directed the student to search for in Wikipedia was. He knew that they would have to work with it, but still spent hours and days supporting the student in exploring and evaluating alternative explanations until they reached a point where the student was willing to consider a mathematical model from the literature which he could not develop by himself. The student's account cited above suggests that he went through an epistemological change.

\section{B. Scaffolding the Understanding of the Explicit Meaning of the Equation}

The mentor constantly prompted and supported an understanding of the explicit meaning of the equation. For example, he attended to the specific meaning of individual variables: "so what do we have here? g we know...". He tediously scaffolded the construction of the student's explicit understanding of complex "sentences" such as the depth expression of the model, which involves a hyperbolic function that the student was not familiar with (Lab, Jan 2, 2017).

146. Men.: /.../ The only thing left for us to examine is this thing. This is the depth expression ((see Figure 1)). Let's just look what this function $((\tanh (\mathrm{x})))$ looks like and then we will understand how

147. Stu.: Do you have a function plotter?

148. Men.: Sure. It's called WolfarmAlfa

$\cdots$

159. Stu.: It's like $X^{3} ! !$ ((referring to the shape of the function))

160. Men.: Ahh, almost, almost. /.../

$\cdots$

162. Men.: And this - it has asymptotes. In 1 and in -1

163. Stu.: Okay. It looks exactly like what we have ((referring to measurement of the velocity at different depths, and his 

164. Men.: This is exactly what we discussed,

realization that from a certain depth the right? Only we need to pay attention that for us it is not X, it's a kind of constant, and the wavelength is also in it.

Note how the mentor called the student's attention to the relevant mathematical features of the function (e.g., asymptotes, turn 162), that was immediately followed by the student's referencing the asymptote in the empirical result. Note also how in turn 164 the mentor called the student's attention to the fact that the hyperbolic function in the model was not a function of a single variable (i.e., "X") but rather a function of a complex expression.

\section{Scaffolding the Understanding of the Implicit Meaning of the Equation}

The following example illustrates scaffolding the understanding of the implicit meaning of the equation. Specifically, here, this involved which components of the model become irrelevant and can be neglected under different conditions. We present an excerpt from the stimulated recall interview with the student on his final report. The student is answering the question why the surface tension expression was neglected in the calculations related to Exp. 2 (Final interview with the student, turn 213).

Right. I'm totally happy about the surface tension $(($ approximation $)) / . . . /$ We saw it was a good approximation while we were doing it. It is a really, really good approximation. All our wavelengths were larger than half a meter, and this approximation worked from $10 \mathrm{~cm} / .$. / It was really smart that we did it. /.../ When we did it ((exp. 2)) I told Shy ((the mentor)) that I was worried about this approximation. He said that we would try to test it on our measurements of velocities for which we knew we had good results. And we tested it and it was fine. So, the approximation was good. But yes, at the beginning I was really worried about this approximation.

The fact that the student explicitly stated how worried he was about the approximation is not surprising. Many students consider approximations as "black magic." The mentor asked the student to calculate the velocity for a series of wavelengths, as well as the ratio between the gravity and the surface tension expressions for different wavelengths. This was a powerful instructional move that demystified this approximation. Clearly this process was meaningful for the student as shown in his decision to include graphs that summarized the results of these calculations in the theoretical background section of his final report. During each stage of the project, a different approximation of the basic model in Figure 1 was employed and student was able to fully justify all of them in the interview.

\section{DISCUSSION}

We presented a case study of a research apprenticeship of high school student. We examined the development of the student's understanding a mathematical equation as a model for a physical phenomenon, his use of that mathematization as a tool and as an object for sense making, and how these were nurtured by the mentor. Our analysis suggests that this process can be paralleled to the development of reading comprehension and that it involves the deciphering of the explicit and implicit meaning of the equation. We also showed that using physics equations in this manner is not intuitive, and requires an epistemological change that needs to be necessitated for the students.

\section{ACKNOWLEDGMENTS}

We deeply the research mentors and students in Acheret Center [16]. This study was supported by the Israel Science Foundation (grant No. 325/17).

[1] Y. Gingras, Hist. Sci. 39, 383 (2001).

[2] M. Steiner, The Applicability of Mathematics as a Philosophical Problem (Harvard University Press, Cambridge, Massachusetts, 1998).

[3] I.A. Halloun, Sci. Educ. 16, 653 (2007).

[4] T.O.B. Odden and R.S. Russ, Sci. Educ. (2018).

[5] A. Gupta and A. Elby, Int. J. Sci. Educ. 33, 2463 (2011).

[6] A.F. Heckler, Int. J. Sci. Educ. 32, 1829 (2010).

[7] U. Leron, in Proc. CERME3 (Bellaria, Italy, 2003), pp. 18.

[8] U. Leron and O. Hazzan, Educ. Stud. Math. 71, 263 (2009).

[9] S. Kapon, Sci. Educ. 101, 165 (2017).

[10] R.S. Russ, R.E. Scherr, D. Hammer, and J. Mikeska, Sci. Educ. 92, 499 (2008).

[11] B.L. Sherin, Cogn. Instr. 19, 479 (2001).

[12] J. Tuminaro and E.F. Redish, Phys. Rev. Spec. Top. - Phys. Educ. Res. 3, 020101 (2007).

[13] S. Kapon, A. Laherto, and O. Levrini, Sci. Educ. 102, 1077 (2018).

[14] D.L. Ball, M.H. Thames, and G. Phelps, J. Teach. Educ. 59, 389 (2008).

[15] D. Basaraba, P. Yovanoff, J. Alonzo, and G. Tindal, Read. Writ. 26, 349 (2013).

[16] http://www.acheret.org.il

[17] S. Kapon, J. Res. Sci. Teach. 53, 1172 (2016).

[18] http://www.surfing-waves.com/waves/wave_terms.htm

[19] S. Kapon and A.A. diSessa, Cogn. Instr. 30, 261 (2012). 